\title{
New Non-Traditional Experimental Techniques to Study Materials at High Pressures
}

\author{
Whitaker, Matthew L.; Rucks, Melinda J.; Sims, Melissa L.; Jaret, Steven J. \\ Department of Geosciences, Stony Brook University, Stony Brook, NY 11794-2100 \\ matthew.whitaker@stonybrook.edu
}

The central purpose to scientific studies is the drive to answer questions to which the answers are currently unknown. Oftentimes, that forces us to think outside the box for ways to go about addressing these unknown problems. Sometimes, thinking outside the box doesn't go far enough and you have to go even further into the realm of insanity to find these new ideas, and throw them at the wall to see what sticks.

Sometimes literally.

There are three main experimental thrusts that will be discussed here:

1) Experiments studying the elasticity of materials using ultra-fast acoustic wave velocity measurements. The system designed and discussed here was $\sim 2$ orders of magnitude faster than other systems of its ilk, and now it has been improved to give better data quality in even shorter collection times, making it $\sim 3$ orders of magnitude faster than all other high pressure ultrasonic interferometry systems currently available.

2) Experiments in the Multi-Anvil Press utilizing fast-advance uniaxial strain application combined with newly developed ultrafast resistive "spike" heating to simulate a proxy for meteoritic impacts.

3) Investigating potential shock-driven phase transitions in silicate materials by pressurizing samples in a Diamond Anvil Cell and then - literally - throwing the cell at the wall and/or hitting it with a hammer. We affectionately call these SMACADAC experiments (Sudden Mechanically Aggressive Compression Action Diamond Anvil Cell).

This presentation will present technical details and some preliminary results of new experiments that we conducted using these new protocols that we have been told were crazy to even attempt. That may be true, but if so, then we posit that while necessity may be the mother of invention, insanity may well then be its father. 